\title{
miR-214 ameliorates acute kidney injury via targeting DKK3 and activating of Wnt/ $\beta$-catenin signaling pathway
}

\author{
Xiaoguang Zhu${ }^{{ }^{*}+}$ (D), Wenwen $\mathrm{Li}^{2 \dagger}$ and Huicong $\mathrm{Li}^{1}$
}

\begin{abstract}
Background: miR-214 was demonstrated to be upregulated in models of renal disease and promoted fibrosis in renal injury independent of TGF- $\beta$ signaling in vivo. However, the detailed role of miR-214 in acute kidney injury (AKI) and its underlying mechanism are still largely unknown.

Methods: In this study, an I/R-induced rat AKI model and a hypoxia-induced NRK-52E cell model were used to study AKI. The concentrations of kidney injury markers serum creatinine, blood urea nitrogen, and kidney injury molecule- 1 were measured. The expressions of miR-214, tumor necrosis factor-a, interleukin (IL)-1 $\beta$, IL-6, were detected by RTqPCR. The protein levels of Bcl-2, Bax, Dickkopf-related protein 3, $\beta$-catenin, c-myc, and cyclinD1 were determined by western blot. Cell apoptosis and caspase 3 activity were evaluated by flow cytometry analysis and caspase 3 activity assay, respectively. Luciferase reporter assay was used to confirm the interaction between miR-214 and Dkk3.

Results: miR-214 expression was induced in ischemia-reperfusion (I/R)-induced AKI rat and hypoxic incubation of NRK-52E cells. Overexpression of miR-214 alleviated hypoxia-induced NRK-52E cell apoptosis while inhibition of miR214 expression exerted the opposite effect. Dkk3 was identified as a target of miR-214. Anti-miR-214 abolished the inhibitory effects of DKK3 knockdown on hypoxia-induced NRK-52E cell apoptosis by inactivation of Wnt/ $\beta$-catenin signaling. Moreover, miR-214 ameliorated AKI in vivo by inhibiting apoptosis and fibrosis through targeting $D k k 3$ and activating Wnt/B-catenin pathway.
\end{abstract}

Conclusion: miR-214 ameliorates AKI by inhibiting apoptosis through targeting Dkk3 and activating Wnt/ $\beta$-catenin signaling pathway, offering the possibility of miR-214 in the therapy of ischemic AKI.

Keywords: miR-214, Acute kidney injury, Dkk3, Wnt/ß-catenin signaling pathway

\section{Background}

Acute kidney injury (AKI), one of the most common complications of major surgical operations and sepsis accompanied by loss of kidney function, can be evaluated through monitoring the changes of renal function markers, such as serum creatinine ( $\mathrm{SCr}$ ), and blood urea nitrogen (BUN) levels $[1,2]$. AKI is associated with higher incidence of mortality and morbidity, prolonged hospital stay, and accelerated chronic kidney disease (CKD) or

\footnotetext{
*Correspondence: zhuxiaoguangzxg@sina.com

${ }^{+}$Xiaoguang Zhu and Wenwen Li contributed equally to this work

${ }^{1}$ Department of Nephrology, Huaihe Hospital of Henan University, No. 8,

Baobei Road, Gulou District, Kaifeng 475000, China

Full list of author information is available at the end of the article
}

end-stage renal disease (ESRD) [3, 4]. Renal ischemiareperfusion (I/R) injury has been demonstrated as the major cause of AKI [5]. The pathophysiological process of AKI is widely known to encompass acute tubular epithelial cell damage, excessive inflammation, acute vascular dysfunction, and fibrosis in response to I/R injury [6]. Although several clinical symptomatic supportive treatments are available, there are still no effective therapies for the treatment of AKI [7].

microRNAs (miRNAs), a class of endogenous, small non-coding RNA molecules with 19-25 nucleotides in length, has emerged as important regulators of gene expression. miRNAs are reported to be involved in a variety of kidney diseases via regulating cell proliferation,

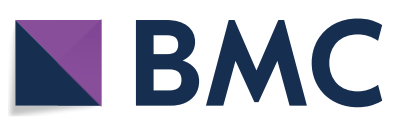

C The Author(s) 2018. This article is distributed under the terms of the Creative Commons Attribution 4.0 International License (http://creativecommons.org/licenses/by/4.0/), which permits unrestricted use, distribution, and reproduction in any medium, provided you give appropriate credit to the original author(s) and the source, provide a link to the Creative Commons license, and indicate if changes were made. The Creative Commons Public Domain Dedication waiver (http://creativecommons.org/ publicdomain/zero/1.0/) applies to the data made available in this article, unless otherwise stated. 
apoptosis, differentiation, and development [8]. A growing body of evidence has highlighted the importance of miRNAs in the pathogenesis of AKI, facilitating the development of diagnostic and therapeutic strategies of AKI $[9,10]$. Previous studies discovered that miR-214 was up-regulated in models of renal disease and promoted fibrosis in renal injury independent of TGF- $\beta$ signaling in vivo $[11,12]$. However, the detailed roles of miR-214 in AKI and its underlying mechanisms are still largely unknown.

Wnt/ $\beta$-catenin signaling pathway, an evolutionarily conserved and key developmental signaling cascade, is implicated in the regulation of various biological processes, including AKI and diabetic nephropathy (DN) $[13,14]$. Wnt/ $\beta$-catenin pathway is reactivated after kidney injury to participate in the development and progression of renal fibrotic lesions in many animal models and human kidney disorders $[15,16]$. Emerging evidence has shown that Wnt/B-catenin signaling plays a pivotal role in promoting kidney repair and regeneration after AKI induced by either I/R or nephrotoxins [17]. Dickkopf (DKK) proteins, an evolutionarily conserved family consisting of DKK1, 2, 3, and 4, have been reported to function as regulators of $\mathrm{Wnt} / \beta$-catenin pathway [18]. They encode secreted proteins that typically antagonize Wnt/ $\beta$-catenin signaling, by inhibiting the Wnt coreceptors LRP5 and 6 [19]. A previous study revealed that Dkk3 could interact with Kremen and LRP with comparable binding energies and block Wnt signaling [20]. microRNA-214 has been uncovered to negatively regulate the $\mathrm{WNT} / \beta$-catenin signaling in human breast cancer and hepatocellular carcinoma [21, 22]. This study was designed to investigate the functions and mechanisms of miR-214 in I/R-induced AKI rat model and hypoxiainduced NRK-52E cell model.

\section{Methods}

\section{Animals}

All animal protocols were performed in accordance with the guidelines for the Care and Use of Laboratory Animals and approved by the Ethics Committee of Henan University of Huaihe Hospital. Healthy male SpragueDawley rats (275-300 g weight, and 11-12 weeks) were obtained from the Beijing Vital River Laboratory Animal Center. Rats were housed under standardized conditions with a constant temperature $\left(23-24{ }^{\circ} \mathrm{C}\right), 50 \%$ humidity, and a light/dark cycle of 12:12 h, and allowed free access to food and water before use in experiments.

\section{Construction of rat AKI model}

The rat AKI model was constructed by renal I/R surgery, as described previously [23]. Briefly, rats were anesthetized by intraperitoneal injection of pentobarbital sodium (50 mg/kg; Sigma-Aldrich) and body temperature of rats were maintained at $37{ }^{\circ} \mathrm{C}$ using heating clamps during surgery. After rats underwent an abdominal incision, bilateral renal pedicles were occluded with a microvascular clamp for $30 \mathrm{~min}$ to induce renal ischemia. The clamps were removed for reperfusion, and then the abdomen was closed in two layers with sutures. For the sham group, rats were subjected to the same surgical procedure, except the renal pedicles were not clamped, and rats without $I / R$ surgery were used as controls. The rats in rat AKI model group and sham group were then administered intraperitoneally with $10 \mathrm{mg} / \mathrm{kg}$ miR-214 mimic (miR-214) or miRNA negative control (miR-con) (GenePharma Co. Ltd, Shanghai, China) in saline. After reperfusion for 12,48 and $72 \mathrm{~h}$, the rats were euthanized and then blood, urine, and kidney tissues were collected.

\section{Renal function measurement}

Serum creatinine and blood urea nitrogen (BUN) in the serum of blood samples were measured using a creatinine assay kit (BioAssay Systems, Hayward, CA) and a Hitachi 7060 automatic biochemistry analyzer (Hitachi, Tokyo, Japan), respectively. The urine concentration of kidney injury molecule-1 (Kim-1) was measured by a commercially available ELISA Kit (Cosmo Bio, Tokyo, Japan).

\section{Cell culture and transfection}

A rat renal proximal tubular cell line (NRK-52E cells) was purchased from American Type Culture Collection (ATCC, Manassas, VA, USA). NRK-52E cells were cultured in Dulbecco's modified Eagle's medium (DMEM; Sigma-Aldrich, St. Louis, MO, USA) containing 10\% fetal bovine serum (FBS; Gibco, Carlsbad, CA, USA), 0.15\% sodium bicarbonate (Sigma-Aldrich), $4 \mathrm{mM}$ L-glutamine (Sigma-Aldrich), and 1\% streptomycin/penicillin (Invitrogen, CA, Carlsbad, USA) in a humidified atmosphere chamber containing $5 \% \mathrm{CO}_{2}$ at $37^{\circ} \mathrm{C}$. For hypoxia experiments, cells were incubated in serum-free medium for $24 \mathrm{~h}$ in a hypoxic chamber (AnaeroPack; Mitsubishi Gas Chemical Co., Inc., Tokyo, Japan) containing $1 \% \mathrm{O}_{2}$, followed by reoxygenation for a further $24 \mathrm{~h}$ to generate hypoxia/reoxygenation $(\mathrm{H} / \mathrm{R})$ cell models. The control group was exposed to normoxic conditions for $48 \mathrm{~h}$.

miR-214 mimic, mimic control (miR-con), miR-214 inhibitor (anti-miR-214), inhibitor control (anti-miRcon), siRNA against $D k k 3$ (si-Dkk3), and siRNA control (si-con) were obtained from GenePharma Co. These oligonucleotides were transfected into NRK-52E cells using Lipofectamine 2000 (Invitrogen).

\section{Determination of caspase 3 activity}

After NRK-52E cells were collected and lysed, and caspase 3 activity was measured using Caspase-3 
Colorimetric Activity Assay Kit (Millipore, Billerica, MA, USA) according to kit instructions. The absorbance at $405 \mathrm{~nm}$ was measured using an ELISA reader (Bio-Rad Laboratories, Inc., Hercules, CA, USA).

\section{Cell apoptosis assay}

Cell apoptosis was analyzed by flow cytometry following the annexin V-fluorescein isothiocyanate (FITC) Apoptosis Detection Kit II (BD Biosciences, San Jose, CA, USA) and propidium iodide (PI) staining according to the manufacturer's instructions. Briefly, cells were collected, washed twice with cold phosphate-buffered saline (PBS), and resuspended in binding buffer. $100 \mu \mathrm{L}$ of cell suspension $\left(2 \times 10^{5}\right.$ cells $)$ was transferred to a 96 -well plate, and incubated with $10 \mu \mathrm{L}$ of Annexin V-FITC and $5 \mu \mathrm{L}$ of propidium iodide (PI) for $15 \mathrm{~min}$ at room temperature in the dark. The apoptotic cells, including FITC-positive and PI-negative cells (early apoptotic), were detected with a FACS flow cytometer (BD Biosciences).

\section{Luciferase reporter assay}

Emerging evidence suggests that Wnt/ $\mathrm{W}$-catenin signaling plays a pivotal role in promoting kidney repair and regeneration after AKI induced by either IR or nephrotoxins [18]. Therefore, the underlying targets, which could regulate $\mathrm{Wnt} / \beta$-catenin signaling, were predicted by Targetscan. Dkk3, an inhibitor of Wnt/ $\beta$-catenin signaling, was selected and investigated in the study. Wild-type or seedregion mutated $D k k 33^{\prime}$ UTR sequences were synthesized and cloned into pmirGLO dual-luciferase miRNA target expression vectors (Promega, Madison, WI, USA), namely $D k k 3-\mathrm{WT}$ and $D k k 3-\mathrm{MUT}$. NRK-52E cells were seeded into 24 -well plates at a density of $1.0 \times 10^{5} /$ well and cotransfected with miR-214 or miR-con, and Dkk3WT or DKK3-MUT, together with pRL-TK renilla plasmids using Lipofectamine 2000 (Invitrogen). At $48 \mathrm{~h}$ posttransfection, cells were harvested for measuring the luciferase activities with the Dual-Luciferase Assay System (Promega). Renilla luciferase activities were detected as a normalization.

\section{Reverse transcription-quantitative polymerase chain reaction (RT-qPCR)}

Total RNA was extracted from resected tissues and cells using TRIzol ${ }^{\circledR}$ reagent (Invitrogen). For the detection of miR-214 expression, $1 \mu \mathrm{g}$ of total RNA was reversely transcribed into cDNA using Taqman ${ }^{\mathrm{TM}}$ microRNA reverse transcription kit (Life Technologies, Gaithersburg, MD, USA) and RT-PCR was performed using TaqMan $^{\text {TM }}$ MicroRNA Assay kit (Applied Biosystems, Foster City, CA, USA). For the measurement of $D k k 3$ mRNA expression, the first-strand cDNA was synthesized from $1 \mu \mathrm{g}$ of total RNA by a High-Capacity cDNA
Archive Kit (Applied Biosystems) and RT-PCR was conducted using a SYBR Premix Ex Taq II Kit (Applied Biosystems). qRT-PCR reaction was carried out using a 7500 Fast Real-Time Sequence detection system (Applied Biosystems). The relative gene expression of miR-214 and $D k k 3$ was calculated using the $2^{-\Delta \Delta C t}$ method. GAPDH and U6 were used as the internal controls for miR-214 and $D k k 3$, respectively.

\section{Western blot}

Cells were harvested and lysed using ice-cold radioimmunoprecipitation assay (RIPA) lysis buffer (Beyotime, Jiangsu, China). Protein concentration was quantified using a Bicinchoninic Acid Protein Assay kit (Sangon Biotech Co., Ltd., Shanghai, China). Total of $10 \mu \mathrm{g}$ protein were subjected to $10 \%$ SDS-PAGE and then transferred onto PVDF membranes (Millipore). The membranes were blocked with $5 \%$ non-fat milk for $1 \mathrm{~h}$ at room temperature and then incubated with primary antibodies fibronectin (BD Biosciences), Bax, Bcl2 , DKK3, $\beta$-catenin, c-myc, cyclin D1 and $\beta$-actin (Cell Signaling Technology, Beverly, MA, USA), followed by further incubation with HRP-labeled secondary antibody (Abcam, Cambridge, MA, USA) for $2 \mathrm{~h}$. The protein signals were visualized using an enhanced chemiluminescence system (ECL ${ }^{\mathrm{TM}}$; Amersham, Little Chalfont, UK).

\section{Statistical analysis}

All experimental data were presented as mean \pm standard deviation (SD). Statistical analyses were performed using SPSS software (version 19.0, IBM, Chicago, IL, USA). Statistical differences between different groups were evaluated by one-way analysis of variance (ANOVA) with Student-Newman-Keuls (SNK) post hoc test and Student's $t$-test. Differences were considered to be statistically significant when $P$ values $<0.05$.

\section{Results \\ Establishment of I/R-induced AKI rat model and hypoxia-induced AKI cell model}

I/R-induced AKI rat model and hypoxia-induced AKI cell model were established to explore the role of miR-214 in AKI progression. The success of I/R-induced rat AKI model was firstly evaluated by measuring the serum levels of SCr, BUN, and urine Kim-1, a potential biomarker in ischemic AKI [24], at $24 \mathrm{~h}$ after surgery. As compared with sham group, the serum levels of SCr (Fig. 1a), BUN (Fig. 1b), and urine Kim-1 (Fig. 1c) were all significantly increased in I/R-induced rat AKI models. Since inflammation and tubular epithelial cell apoptosis are the major features of AKI [25], the effectiveness of hypoxia-induced NRK-52E cell model was further assessed by detecting the expression of inflammatory factors including tumor 

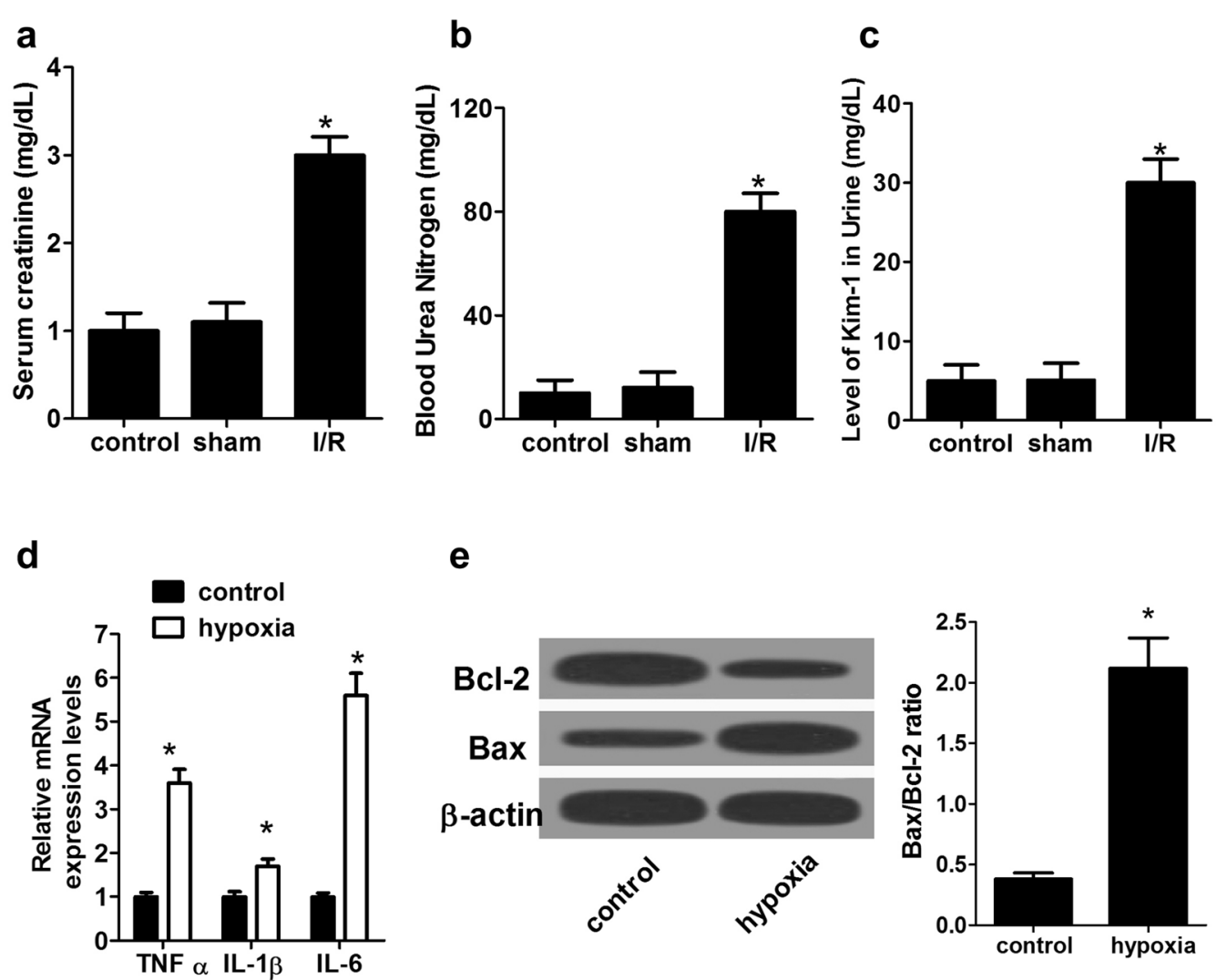

$\mathbf{e}$
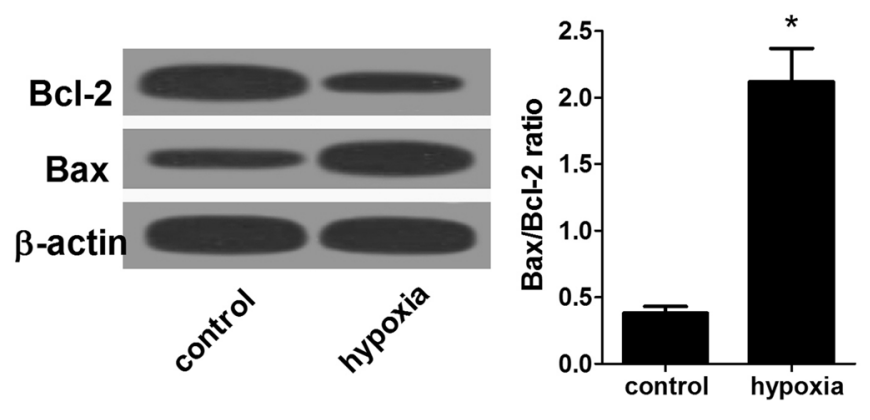

Fig. 1 Evaluation of rat AKI model following I/R surgery and NRK-52E cell model following hypoxia treatment. The serum levels of $\mathrm{SCr}$ (a), BUN (b), and urine Kim-1 (c) in I/R-induced rat AKI models at $24 \mathrm{~h}$ after surgery were measured. $\mathbf{d}$ The mRNA expressions of TNF-a, IL-1 $\beta$, and IL-6 in hypoxia-induced NRK-52E cell I/R model were detected by qRT-PCR. e The protein levels of BCl-2 and Bax in hypoxia-induced NRK-52E cell I/R models were assessed by western blot. $n=6$ rats/group. ${ }^{*} P<0.05$

necrosis factor (TNF)- $\alpha$, interleukin (IL)-1 $\beta$, and IL-6, and examining apoptosis [26]. qRT-PCR results demonstrated that mRNA expressions of TNF- $\alpha$, IL-1 $\beta$, and IL- 6 were markedly promoted under hypoxia compared with cells under normoxic conditions (Fig. 1d). Also, western blot analyses demonstrated that hypoxia-induced NRK$52 \mathrm{E}$ cells showed an obvious decrease of $\mathrm{Bcl}-2$ protein level and an apparent increase of Bax level (Fig. 1e), suggesting the emergence of apoptosis. All these results indicated the successful construction of I/R-induced rat AKI models and hypoxia-induced cell I/R model.

\section{Overexpression of miR-214 alleviated hypoxia-induced apoptosis in NRK-52E cells}

The expression of miR-214 in kidney tissues of I/Rinduced rat AKI models was analyzed by qRT-PCR and the in vivo results demonstrated that miR-214 expression was aberrantly upregulated in I/R-induced rat AKI models after 12,48 and $72 \mathrm{~h}$ of reperfusion compared to that in sham group (Fig. 2a). The expression of miR-214 during hypoxic incubation of cultured NRK-52E cells was also evaluated by qRT-PCR and the in vitro results demonstrated that miR-214 expression was increased at $0-6 \mathrm{~h}$, and reached the highest at $6 \mathrm{~h}$ after hypoxia treatment (Fig. 2b). These results indicated that miR-214 was induced during renal $\mathrm{I} / \mathrm{R}$ in kidney tissues and hypoxia in cultured renal proximal tubular cells. To determine the role of miR-214 in AKI, loss- and gain-of-function experiments were performed in hypoxia-induced NRK-52E cells by transfecting with miR-214 or anti-miR-214. qRTPCR results demonstrated that miR-214 transfection dramatically promoted miR-214 expression and introduction of anti-miR-214 significantly lowered miR-214 expression (Fig. 2c). Flow cytometry analysis showed that exogenous expression of miR-214 led to an obvious suppression on hypoxia-induced apoptosis in NRK-52E cells, while miR-214 inhibitor resulted in a marked enhancement of apoptotic rate in NRK-52E cells during hypoxia treatment (Fig. 2d, e). Meanwhile, western blot results indicated that forced expression of miR-214 promoted Bcl-2 level and repressed Bax level, while down-regulation of miR-214 elicited the opposite effects on Bcl-2 and Bax 

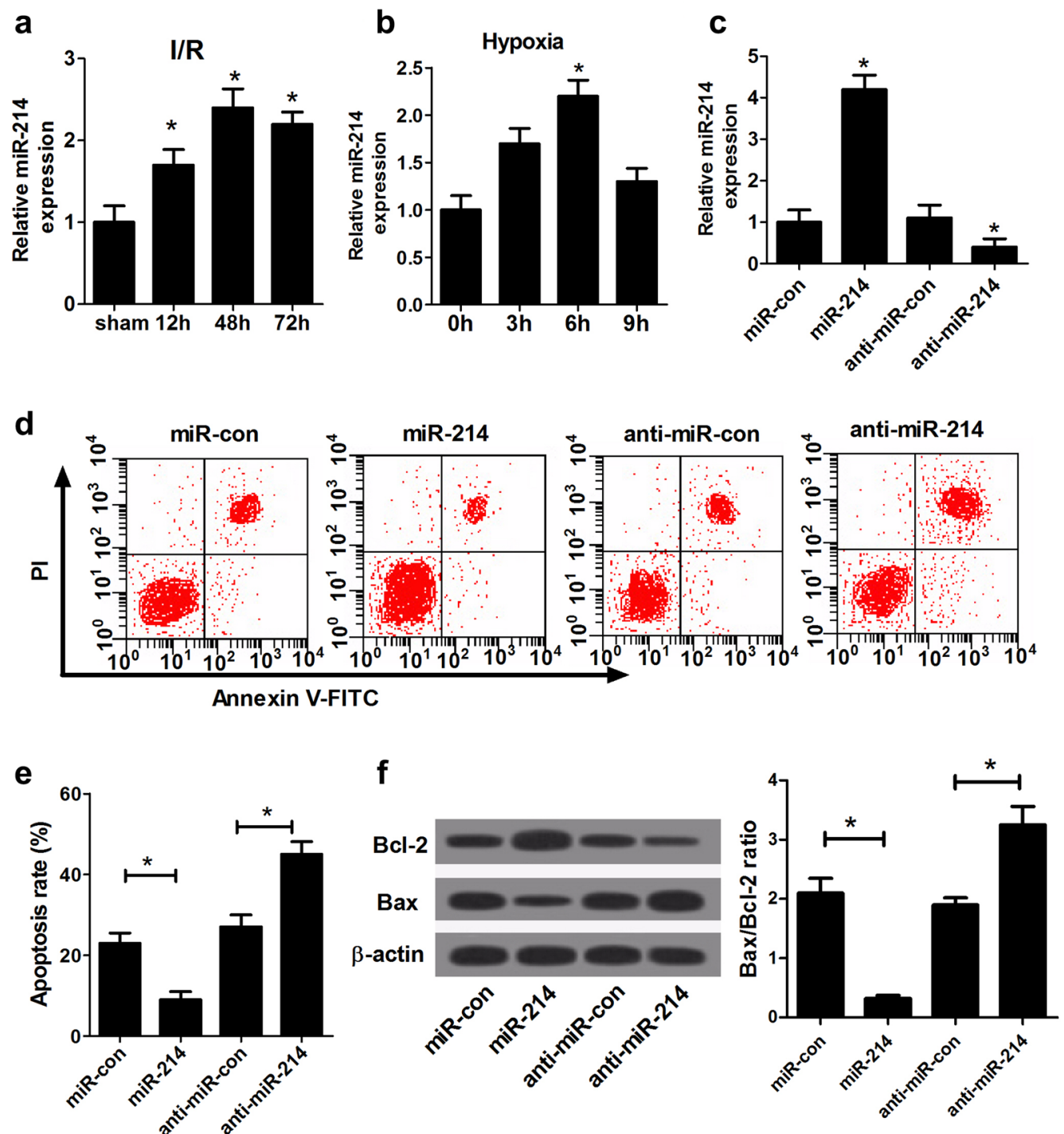

Fig. 2 miR-214 attenuated NRK-52E cell apoptosis under hypoxia. a qRT-PCR analysis of miR-214 expression in kidney cortex of $\mathrm{l} / \mathrm{R}$-induced rat AKI models after 12,48 and $72 \mathrm{~h}$ of reperfusion. $n=6$ rats/group. $\mathbf{b}$ qRT-PCR analysis of miR-214 expression at $0,3 \mathrm{~h}, 6 \mathrm{~h}$, and $9 \mathrm{~h}$ during hypoxic incubation of cultured NRK-52E cells. c qRT-PCR analysis of miR-214 expression in NRK-52E cells after transfection with miR-214, anti-miR-214, or matched controls. d-e Flow cytometry was used to analyze the apoptotic rate of NRK-52E cells after transfection with miR-214, anti-miR-214, or respective controls during hypoxia incubation. $\mathbf{f}$ Western blot was performed to detect the protein levels of Bcl-2 and Bax in NRK-52E cells after introduction with miR-214, anti-miR-214, or corresponding controls under hypoxia. Each experiment was independently repeated 3 times. ${ }^{*} P<0.05$

expression (Fig. 2f). Therefore, these results suggested that miR-214 induced in I/R may exert a protective effect on tubular cell injury by inhibiting apoptosis.

\section{miR-214 suppressed DKK3 expression in NRK-52E cells}

Bioinformatics analyses predicted that $3^{\prime} \mathrm{UTR}$ of $D k k 3$ contained some conserved binding sequences of miR-214 (Fig. 3a). To confirm whether $D k k 3$ was a target of miR214 in NRK-52E cells, either wild-type or mutated $3^{\prime} U T R$ sequences of $D k k 3$ were cloned into luciferase reporter plasmids. Luciferase reporter assay results proved that overexpression of miR-214 significantly restrained the relative luciferase activity of DKK3-WT, but exerted no inhibitory effect on the relative luciferase activity of Dkk3-MUT (Fig. 3b). Additionally, endogenous DKK3 expression at mRNA and protein levels in NRK-52E cells transfected with miR-214 or anti-miR-214 were evaluated by qRT-PCR and western blot. As displayed in Fig. 3c-e, DKK3 expression at both mRNA and protein levels was prominently reduced following ectopic expression of 


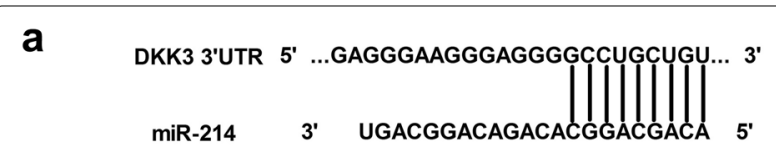

Mutant DKK3 3'UTR 5' ...GAGgGaAggaggGUAAGUAGUG... 3'
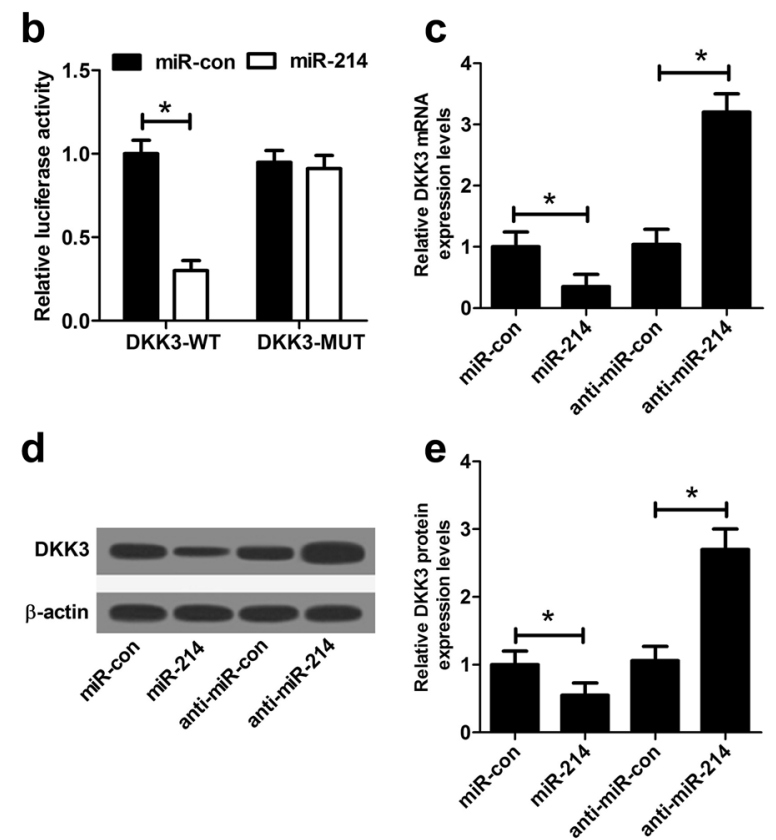

Fig. 3 Dkk3 was a target of miR-214 in NRK-52E cells. a The predictive wild-type miR-214 binding sites in the $3^{\prime} U T R$ of Dkk3 and the corresponding mutant binding sites were displayed. $\mathbf{b}$ The relative luciferase activity was measured by luciferase reporter assay after NRK-52E cells were cotransfected with Dkk3-WT or Dkk3-MUT and miR-214 or miR-con. The mRNA (c) and protein $(\mathbf{d}$, e) levels of DKK3 were examined by qRT-PCR and western blot in NRK-52E cells introduced with miR-214, anti-miR-214, or matched controls. Each experiment was independently repeated 3 times. ${ }^{*} P<0.05$

miR-214 and enhanced by miR-214 inhibitor. Together, these data revealed that miR-214 targeted $D k k 3$ by binding to the 3'UTR of $D k k 3$ in NRK-52E cells.

\section{Anti-miR-214 abolished the inhibitory effects of DKK3 knockdown on hypoxia-induced apoptosis in NRK-52E cells by inactivation of $\mathrm{Wnt} / \boldsymbol{\beta}$-catenin signaling}

Firstly, the protein expression of DKK3 was analyzed in I/R-induced rat AKI models and hypoxia-induced NRK$52 \mathrm{E}$ cell model. The results displayed that the protein level of DKK3 in kidney tissues of I/R-induced rat AKI models was increased after $30 \mathrm{~min}$ of ischemia and 12 or $48 \mathrm{~h}$ of reperfusion when compared with sham group (Fig. 4a). Moreover, $D k k 3$ expression was also enhanced in NRK$52 \mathrm{E}$ cells after hypoxia treatment, and the increase was most significant at $6 \mathrm{~h}$ after hypoxic treatment (Fig. 4b).
To explore the role of DKK3 in miR-214-mediated renal protection, NRK-52E cells were transfected with siDKK3 alone or along with anti-miR-214. As expected, DKK3 level was markedly reduced by introduction of si-DKK3, but DKK3 expression was notably restored by cotransfection with anti-miR-214 (Fig. 4c). Flow cytometry analyses demonstrated that DKK3 knockdown significantly blocked hypoxia-induced apoptosis in NRK$52 \mathrm{E}$ cells, while this inhibitory effect was weakened after down-regulating miR-214 (Fig. 4d). Similarly, caspase 3 activity assay revealed that caspase 3 activity was strikingly reduced by DKK3 knockdown, however, inhibition of miR-214 relieved si-DKK3-induced decrease of caspase 3 activity in NRK-52E cells following hypoxia treatment (Fig. 4e). In line with the above results, DKK3 knockdown markedly increased Bcl-2 expression and decreased Bax level, which was substantially abrogated by miR-214 repression (Fig. 4f). DKK3 has been reported to function as a negative regulator of $\mathrm{Wnt} / \beta$-catenin pathway. Thus, the effects of DKK3 knockdown and miR214 inhibition on $\mathrm{Wnt} / \beta$-catenin pathway were analyzed by detecting the protein levels of $\beta$-catenin and its downstream genes (c-myc, and cyclin D1). As shown in Fig. 4g, the protein levels of $\beta$-catenin, $c$-myc, and cyclin D1 were significantly increased in NRK-52E cells after transfection with si-DKK3 compared with si-NC group (Fig. 4g). However, cotransfection with si-DKK3 and anti-miR-214 significantly abated the increase of the protein levels of $\beta$-catenin, c-myc, and cyclin D1 induced by si-DKK3, suggesting that miR-214 suppression abolished DKK3 knockdown-induced inhibition on the Wnt/ $\beta$-catenin pathway. Moreover, the mRNA expressions and concentrations of TNF- $\alpha$, IL-1 $\beta$, and IL- 6 were significantly decreased in NRK-52E cells after transfection with siDKK3 compared with si-NC group (Fig. 4h, i). Together, it is concluded that DKK3 knockdown could protect against tubular cell injury by blocking Wnt/ $\beta$-catenin pathway, and this effect was mediated by miR-214.

miR-214 ameliorated AKI in vivo by inhibiting apoptosis and fibrosis via targeting Dkk3 and activating Wnt/ $\beta$-catenin pathway

To confirm the molecular mechanism of miR-214 involved in the pathogenesis of AKI in vivo, miR-214 or miR-con was intraperitoneally injected into mice, followed by ischemic surgery. Then the renal function was assessed through monitoring the serum levels of $\mathrm{SCr}$ and BUN, and urine Kim-1. qRT-PCR showed that miR-214 expression was significantly improved by miR-214 injection in I/R-induced AKI rats versus that in sham group (Fig. 5a). As compared with sham group, the concentrations of SCr (Fig. 5b), BUN (Fig. 5c), and urine Kim-1 (Fig. 5d) were all reduced following miR-214 treatment in 

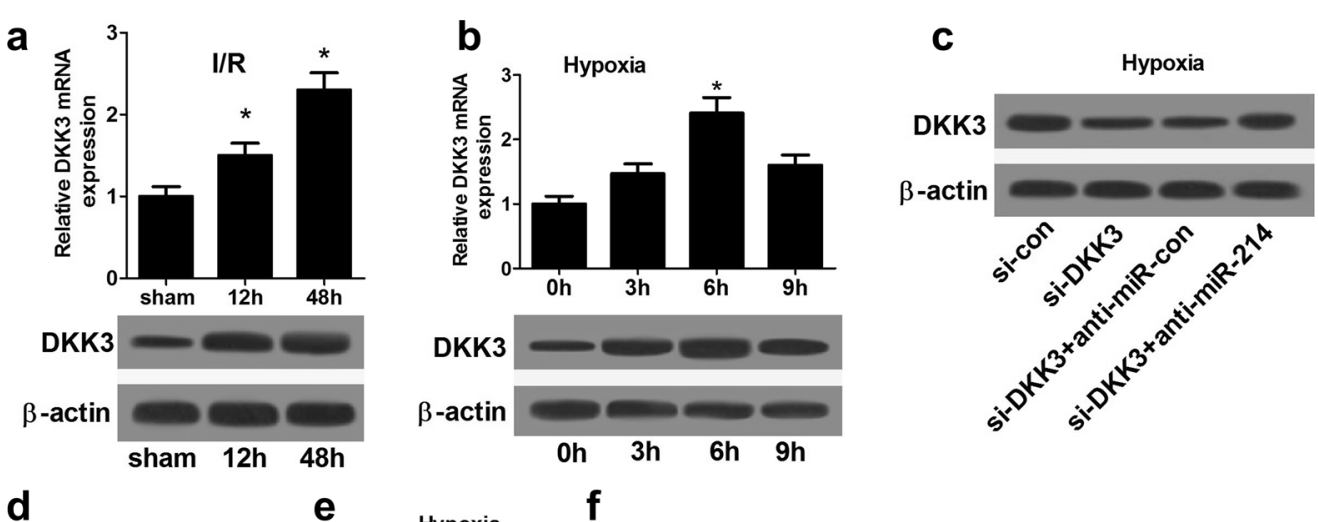

d
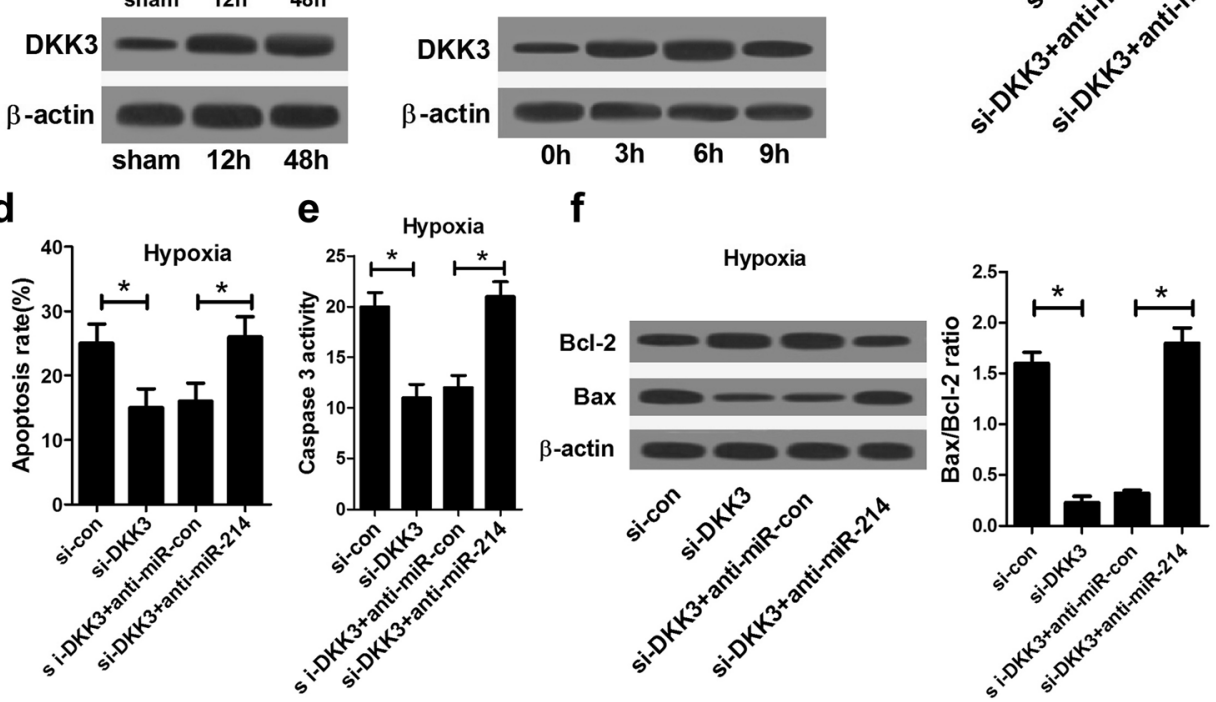
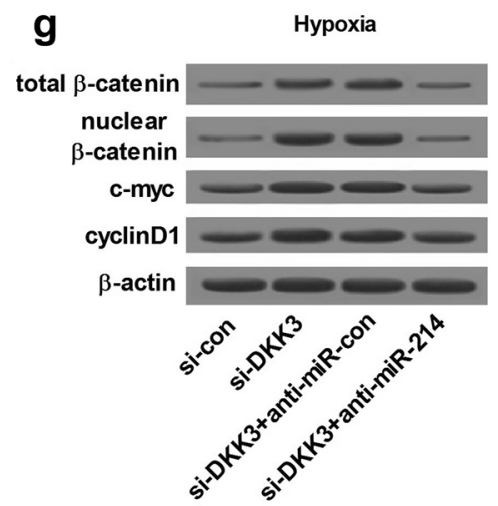

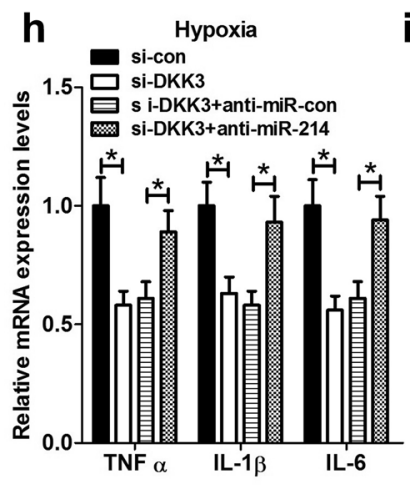

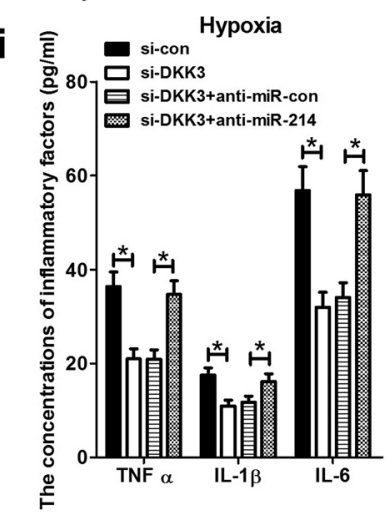

Fig. 4 Anti-miR-214 abolished the inhibitory effect of DKK3 knockdown on hypoxia-induced apoptosis in NRK-52E cells by inactivation of Wnt/B-catenin pathway. Western blot analysis of DKK3 level in kidney tissues of I/R-induced rat AKI models after 30 min of ischemia and 12 or $48 \mathrm{~h}$ of reperfusion (a) and cultured NRK-52E cells after hypoxia treatment (b). NRK-52E cells transfected with either si-DKK3 alone or combined with anti-miR-214 were incubated under hypoxia, followed by detection of DDK3 protein expression (c), apoptosis (d), caspase 3 activity (e), Bcl-2 and Bax protein levels (f), $\beta$-catenin, c-myc, cyclin D1 expression (g) mRNA expressions of TNF-a, IL-1 $\beta$ and IL- 6 (h), and the concentrations of TNF-a, IL-1 $\beta$ and IL-6 (i). Each experiment was independently repeated 3 times. ${ }^{*} P<0.05$

I/R-induced AKI rats, suggesting the amelioration of AKI by miR-214. In addition, forced expression of miR-214 enhanced Bcl-2 level, reduced Bax level and decreased fibronectin protein level in I/R-induced rat AKI model relative to that in sham group (Fig. 5e), suggesting that overexpression of miR-214 inhibited apoptosis and fibrosis in I/R-induced rat AKI model. Furthermore, western blot analysis demonstrated that exogenous expression of miR-214 inhibited the protein level of DKK3 and promoted $\beta$-catenin, c-myc, and cyclin D1 levels in I/Rinduced rat AKI model when compared with that in sham group (Fig. 5f), suggesting that miR-214 activated the Wnt/ $\beta$-catenin pathway by targeting $D k k 3$. These data demonstrated that miR-214 ameliorated AKI in vivo by repressing apoptosis through targeting $D k k 3$ and activating $\mathrm{Wnt} / \beta$-catenin pathway. 

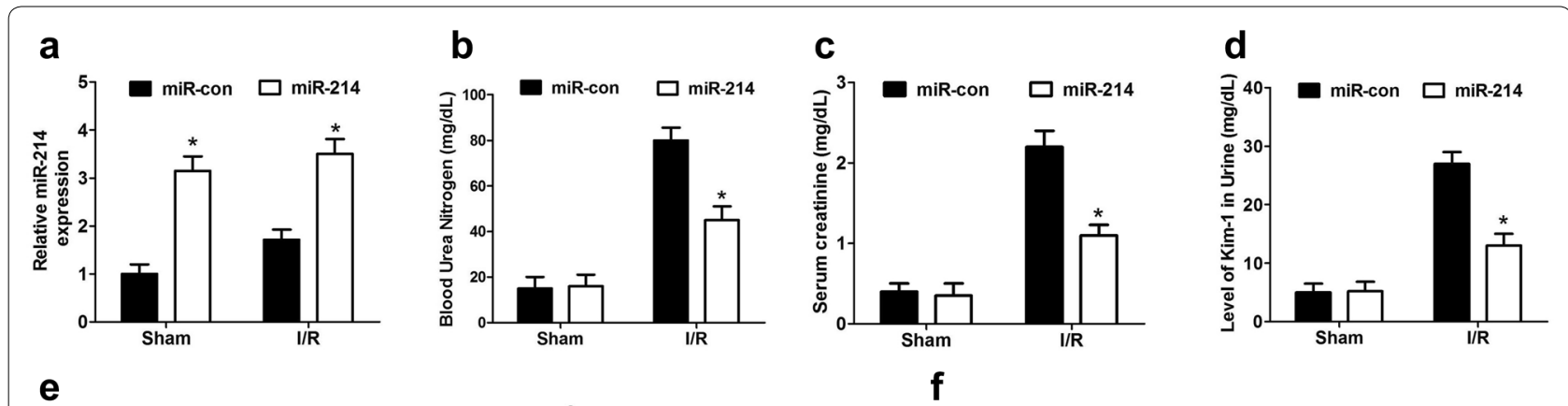

e
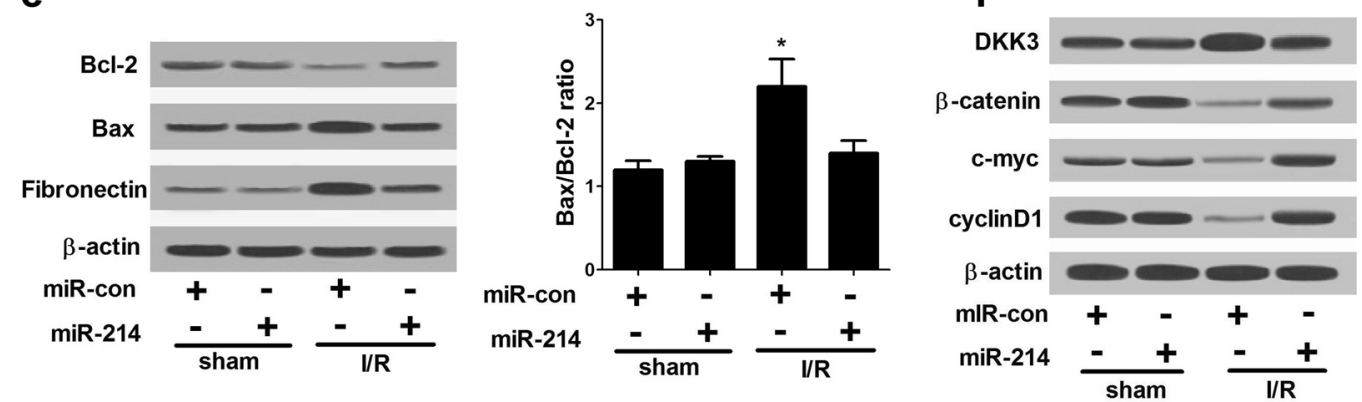

Fig. 5 miR-214 protected against AKl in vivo through targeting Dkk3 and activating Wnt/ $\beta$-catenin pathway. miR-214 or miR-con was intraperitoneally injected into mice, followed by ischemic surgery. a qRT-PCR analysis of miR-214 expression in I/R-induced rat AKI model and sham mice. The serum levels of BUN (b), SCr (c), and Urine Kim-1 (d) level in I/R-induced rat AKI model and sham mice. e Western blot analysis of BCl-2, Bax and fibronectin in I/R-induced rat AKI model and sham group. $\mathbf{f}$ The protein levels of DKK3, $\beta$-catenin, c-myc, and cyclin D1 in I/R-induced rat AKI model and sham group. Each experiment was independently repeated 3 times. ${ }^{*} P<0.05$

\section{Discussion}

In the present study, the I/R-induced rat AKI model and hypoxia-induced NRK-52E cell model were established to explore the significance of miR-214 in the development of AKI and its underlying mechanisms. It is found that miR214 was induced in I/R-induced AKI rats and hypoxiainduced renal proximal tubular cells, and overexpression of miR-214 alleviated hypoxia-induced NRK-52E cell apoptosis. $D k k 3$ was identified as a target of miR-214 in NRK-52E cells. Moreover, down-regulation of miR-214 abated the inhibitory effects of DKK3 knockdown on hypoxia-induced NRK-52E cell apoptosis by inactivation of $\mathrm{Wnt} / \beta$-catenin pathway. In vivo experiments further disclosed that miR-214 ameliorated AKI in vivo by suppressing apoptosis and fibrosis through targeting $D k k 3$ and activating Wnt/ $\beta$-catenin pathway.

Accumulating evidence elucidates that dysregulated miRNAs are implicated in the pathogenesis of kidney injury and renal fibrosis [27, 28]. For instance, transfer of miR-489 expression from human cord blood endothelial colony-forming cell-derived exosomes reduced ischemic kidney injury by targeting phosphatase and tensin homolog (PTEN) through activation of Akt pathway [29]. miR-107 induced TNF- $\alpha$ secretion by targeting Dualspecificity phosphatase 7(DUSP7) in endothelial cells, triggering tubular cell injury in septic AKI [30]. miR-16 transactivated by CCAAT enhancer binding protein beta $(C / E B P-\beta)$ attenuated kidney function and increased kidney apoptosis after I/R injury [31]. Several studies have focused on comprehensive investigations into the biological functions of miR-214. miR-214 expression was up-regulated in monocytes of patients with chronic kidney disease [32]. Notably, previous documents illustrated that miR-214 protected cells from hypoxia/reoxygenation $(\mathrm{H} / \mathrm{R})$ induced damage, and attenuated $\mathrm{I} / \mathrm{R}$-induced cardiac myocardial injury and I/R-induced cardiac myocyte apoptosis by modulating PTEN expression through activation of PI3K/Akt pathway [33, 34]. In addition, miR214 and miR-21 were consistently modulated during renal injury [11]. Therefore, the biological roles of miR214 and miR-21 may be also consistent in renal injury. Up-regulation of miR-21 by ghrelin ameliorated I/Rinduced AKI by inhibiting the inflammatory responses and renal tubular epithelial cell apoptosis via stimulating PI3K/Akt pathway [35]. Besides, miR-21 overexpression suppressed kidney cell apoptosis induced by sepsis in AKI by affecting PTEN/PI3K/AKT signaling pathway [36]. In the present study, the rat AKI model was successfully established as demonstrated by the increased serum levels of SCr, BUN, and urine Kim-1. NRK-52E cell $\mathrm{I} / \mathrm{R}$ model was also constructed accompanied by the enhanced expressions of inflammatory factors and 
the emergence of apoptosis. Also, miR-214 expression was induced in ischemic AKI and hypoxic incubation of kidney cells, similar with increased miR-489 expression in renal tubular cells after ischemic AKI [37]. Loss- and gain-of-function experiments demonstrated that miR214 alleviated hypoxia-induced NRK-52E cell apoptosis, indicating that miR-214 ameliorated AKI by inhibiting apoptosis.

Wnt $/ \beta$-catenin pathway is transiently activated after $\mathrm{AKI}$, and identified as a protective response to minimize cell damage by facilitating tubular repair and regeneration [18]. For instance, Wnt agonist improved renal regeneration and function while attenuated inflammation and oxidative stress in the kidneys after I/R [38]. Administration with erythropoietin improved the kidney against I/R injury with activation of Wnt/B-catenin pathway [39]. However, the exaggerated and sustained activation of $\mathrm{Wnt} / \beta$-catenin pathway may contribute to the transition of AKI to chronic kidney disease (CKD) [40]. Through interacting with the different proteins of Wnt signaling cascade, DKK3, a Wnt/ $\beta$-catenin signaling antagonist, has been demonstrated to be implicated in numerous biological processes, including embryonic development and tumorigenesis [41]. DKK3 was reported to suppress the $\mathrm{Wnt} / \beta$-catenin signaling in proximal tubular epithelial cells (PTECs), therefore inducing tubular cell death in proteinuric nephropathy [42]. In nucleus, $\beta$-catenin could bind to TCF/LEF and stimulate related downstream genes, including c-myc and cyclinD1 [43]. To explore the molecular mechanism by which miR-214 inhibited hypoxia-induced NRK-52E cell apoptosis, the potential targets of miR-214 were predicted. Luciferase reporter assay, RT-qPCR and western blot analyses demonstrated that $D k k 3$ was a target of miR-214 and miR-214 suppressed DKK3 expression, which was also induced in ischemic AKI and hypoxia-induced NRK-52E cells. Mechanistic analysis demonstrated that miR-214 inhibition reversed the suppressive effect of DKK3 knockdown on hypoxia-induced NRK-52E cell apoptosis by inactivation of $\mathrm{Wnt} / \beta$-catenin signaling in vitro, while miR-214 ameliorated AKI in vivo through decreasing apoptosis by targeting DKK3 and activating Wnt/ $\beta$-catenin pathway. Although the data suggest that overexpression of miR214 may be protective immediately after $I / R$, the longterm consequences of miR-214 up-regulation remain unclear. Previous studies showed that persistent gain of miR-214 in tubules leads to pro-inflammatory and profibrotic phenotypes, which may contribute to chronic kidney disease after AKI. Optimal dosing and timing of delivery of miR-214 mimics after AKI should therefore be further investigated to mitigate such potential late adverse effects.

\section{Conclusion}

In conclusion, this study demonstrated that miR-214 was induced during ischemic AKI and hypoxic incubation of kidney cells. Upon induction, miR-214 may target DKK3 to suppress apoptosis via activation of $\mathrm{Wnt} / \beta$-catenin pathway during ischemic or hypoxic renal damage. Therefore, miR-214 may serve as a new therapeutic target for the prevention and treatment of AKI.

\section{Additional file}

Additional file 1. The original data of western blot in this study.

\section{Abbreviations}

AKI: acute kidney injury; Kim-1: kidney injury molecule-1; SCr: serum creatinine; BUN: blood urea nitrogen; TNF-a: tumor necrosis factor-a; ESRD: end-stage renal disease; CKD: chronic kidney disease; miRNAs: microRNAs; DN: diabetic nephropathy; FBS: fetal bovine serum.

\section{Authors' contributions}

$X Z$ and $\mathrm{HL}$ designed and performed the experiments. $X Z$ and $W L$ analyzed the data and wrote the manuscript. HL supervised the study and reviewed the manuscript. All authors read approved the final manuscript.

\section{Author details}

${ }^{1}$ Department of Nephrology, Huaihe Hospital of Henan University, No. 8, Baobei Road, Gulou District, Kaifeng 475000, China. ${ }^{2}$ Department of Cardiology, Huaihe Hospital of Henan University, Kaifeng 475000, China.

\section{Acknowledgements}

Not applicable.

\section{Competing interests}

The authors declare that they have no competing interests.

\section{Availability of data and materials}

Some of the data were available in Additional file 1.

\section{Consent for publication \\ Not applicable.}

\section{Ethics approval and consent to participate}

All animal protocols were performed in accordance with the guidelines for the Care and Use of Laboratory Animals and approved by the Ethics Committee of Henan University of Huaihe Hospital.

\section{Funding}

None.

\section{Publisher's Note}

Springer Nature remains neutral with regard to jurisdictional claims in published maps and institutional affiliations.

Received: 13 March 2018 Accepted: 28 August 2018

Published online: 04 September 2018

\section{References}

1. Singbartl K, Kellum JA. AKI in the ICU: definition, epidemiology, risk stratification, and outcomes. Kidney Int. 2012;81:819-25.

2. Bellomo R, Kellum JA, Ronco C. Acute kidney injury. Lancet. 2012:380:756-66. 
3. Chawla LS, Eggers PW, Star RA, Kimmel PL. Acute kidney injury and chronic kidney disease as interconnected syndromes. N Engl J Med. 2014;371:58-66

4. Chertow GM, Burdick E, Honour M, Bonventre JV, Bates DW. Acute kidney injury, mortality, length of stay, and costs in hospitalized patients. J Am Soc Nephrol. 2005;16:3365-70.

5. Bonventre JV, Yang L. Cellular pathophysiology of ischemic acute kidney injury. J Clin Invest. 2011;121:4210-21.

6. Li YF, Jing Y, Hao J, Frankfort NC, Zhou X, Shen B, et al. MicroRNA-21 in the pathogenesis of acute kidney injury. Protein Cell. 2013;4:813-9.

7. Arai S, Kitada K, Yamazaki T, Takai R, Zhang X, Tsugawa Y, et al. Apoptosis inhibitor of macrophage protein enhances intraluminal debris clearance and ameliorates acute kidney injury in mice. Nat Med. 2016:22:183-93.

8. Jaswani P, Prakash S, Dhar A, Sharma RK, Prasad N, Agrawal S. MicroRNAs involvement in renal pathophysiology: a bird's eye view. Indian J Nephrol. 2017;27:337-41.

9. Zhou P, Chen Z, Zou Y, Wan X. Roles of non-coding RNAs in acute kidney injury. Kidney Blood Press Res. 2016;41:757-69.

10. Liu Z, Wang S, Mi QS, Dong Z. MicroRNAs in pathogenesis of acute kidney injury. Nephron. 2016:134:149-53.

11. Denby L, Ramdas V, McBride MW, Wang J, Robinson H, McClure J, et al. miR-21 and miR-214 are consistently modulated during renal injury in rodent models. Am J Pathol. 2011;179:661-72.

12. Denby L, Ramdas V, Lu R, Conway BR, Grant JS, Dickinson B, et al. MicroRNA-214 antagonism protects against renal fibrosis. J Am Soc Nephrol. 2014;25:65-80.

13. Clevers $H$, Nusse R. Wnt/ß-catenin signaling and disease. Cell. 2012;149:1192-205

14. Uhlenhaut $\mathrm{NH}$, Treier $\mathrm{M}$. Transcriptional regulators in kidney disease: gatekeepers of renal homeostasis. Trends Genet. 2008;24:361-71.

15. Kawakami T, Ren S, Duffield JS. Wnt signalling in kidney diseases: dual roles in renal injury and repair. J Pathol. 2013;229:221-31.

16. Tan RJ, Zhou D, Zhou L, Liu Y. Wnt/ $\beta$-catenin signaling and kidney fibrosis. Kidney Int Suppl. 2014;4:84-90.

17. Zhou D, Li Y, Lin L, Zhou L, Igarashi P, Liu Y. Tubule-specific ablation of endogenous $\beta$-catenin aggravates acute kidney injury in mice. Kidney Int. 2012;82:537-47

18. Zhou S, Zhu Y, Mashrah M, Zhang X, He Z, Yao Z, et al. Expression pattern of DKK3, dickkopf WNT signaling pathway inhibitor 3, in the malignant progression of oral submucous fibrosis. Oncol Rep. 2017;37:979-85.

19. Niehrs C. Function and biological roles of the Dickkopf family of Wnt modulators. Oncogene. 2006:25:7469-81.

20. Mohammadpour H, Pourfathollah AA, Nikougoftar Zarif M, Khalili S. Key role of Dkk3 protein in inhibition of cancer cell proliferation: an in silico identification. J Theor Biol. 2016;393:98-104.

21. Yi SJ, Li LL, Tu WB. MiR-214 negatively regulates proliferation and WNT/ $\beta$-catenin signaling in breast cancer. Eur Rev Med Pharmacol Sci. 2016;20:5148-54

22. Xia H, Ooi LLPJ, Hui KM. MiR-214 targets $\beta$-catenin pathway to suppress invasion, stem-like traits and recurrence of human hepatocellular carcinoma. PLoS ONE. 2012;7:e44206.

23. Zager RA, Johnson AC, Hanson SY, Lund S. Ischemic proximal tubular injury primes mice to endotoxin-induced TNF-alpha generation and systemic release. Am J Physiol Renal Physiol. 2005;289:F289-97.

24. Han WK, Bailly V, Abichandani $R$, Thadhani $R$, Bonventre JV. Kidney injury molecule-1 (KIM-1): a novel biomarker for human renal proximal tubule injury. Kidney Int. 2002;62:237-44.

25. Manne ND, Arvapalli R, Nepal N, Shokuhfar T, Rice KM, Asano S, et al. Cerium oxide nanoparticles attenuate acute kidney injury induced by intra-abdominal infection in Sprague-Dawley rats. J Nanobiotechnol. 2015;13:75.

26. Ratliff BB, Rabadi MM, Vasko R, Yasuda K, Goligorsky MS. Messengers without borders: mediators of systemic inflammatory response in AKI. J Am Soc Nephrol. 2013:24:529-36.

27. Patel V, Noureddine L. MicroRNAs and fibrosis. Curr Opin Nephrol Hypertens. 2012:21:410-6.

28. Fan PC, Chen CC, Chen YC, Chang YS, Chu PH. MicroRNAs in acute kidney injury. Hum Genomics. 2016:10:29.

29. Vinas JL, Burger D, Zimpelmann J, Haneef R, Knoll W, Campbell P, et al. Transfer of microRNA-486-5p from human endothelial colony forming cell-derived exosomes reduces ischemic kidney injury. Kidney Int 2016;90:1238-50

30. Wang S, Zhang Z, Wang J, Miao H. MiR-107 induces TNF-alpha secretion in endothelial cells causing tubular cell injury in patients with septic acute kidney injury. Biochem Biophys Res Commun. 2017:483:45-51.

31. Chen HH, Lan YF, Li HF, Cheng CF, Lai PF, Li WH, et al. Urinary miR-16 transactivated by C/EBPbeta reduces kidney function after ischemia/ reperfusion-induced injury. Sci Rep. 2016;6:27945.

32. Li LM, Hou DX, Guo YL, Yang JW, Liu Y, Zhang CY, et al. Role of microRNA214-targeting phosphatase and tensin homolog in advanced glycation end product-induced apoptosis delay in monocytes. J Immunol. 2011;186:2552-60.

33. Aurora AB, Mahmoud Al, Luo X, Johnson BA, van Rooij E, Matsuzaki S, et al. MicroRNA-214 protects the mouse heart from ischemic injury by controlling $\mathrm{Ca}^{2+}$ overload and cell death. J Clin Invest. 2012;122:1222-32.

34. Wang X, Ha T, Hu Y, Lu C, Liu L, Zhang X, et al. MicroRNA-214 protects against hypoxia/reoxygenation induced cell damage and myocardial ischemia/reperfusion injury via suppression of PTEN and Bim1 expression. Oncotarget. 2016;7:86926-36.

35. Zhang W, Shu L. Upregulation of miR-21 by ghrelin ameliorates ischemia/ reperfusion-induced acute kidney injury by inhibiting inflammation and cell apoptosis. DNA Cell Biol. 2016;35:417-25.

36. Fu D, Dong J, Li P, Tang C, Cheng W, Xu Z, et al. MiRNA-21 has effects to protect kidney injury induced by sepsis. Biomed Pharmacother. 2017:94:1138-44

37. Wei Q, Liu Y, Liu P, Hao J, Liang M, Mi QS, et al. MicroRNA-489 induction by hypoxia-inducible factor-1 protects against ischemic kidney injury. J Am Soc Nephrol. 2016;27:2784-96.

38. Kuncewitch M, Yang WL, Corbo L, Khader A, Nicastro J, Coppa GF, et al. WNT agonist decreases tissue damage and improves renal function after ischemia-reperfusion. Shock. 2015:43:268-75.

39. Chen X, Wang CC, Song SM, Wei SY, Li JS, Zhao SL, et al. The administration of erythropoietin attenuates kidney injury induced by ischemia/reperfusion with increased activation of Wnt/ $\beta$-catenin signaling. J Formos Med Assoc. 2015;114:430-7.

40. Xiao L, Zhou D, Tan RJ, Fu H, Zhou L, Hou FF, et al. Sustained activation of Wnt/B-catenin signaling drives AKI to CKD progression. J Am Soc Nephrol. 2016:27:1727-40.

41. Wang Z, Lin L, Thomas DG, Nadal E, Chang AC, Beer DG, et al. The role of Dickkopf-3 overexpression in esophageal adenocarcinoma. J Thorac Cardiovasc Surg. 2015;150:377-85.

42. Wong DW, Yiu WH, Wu HJ, Li RX, Liu Y, Chan KW, et al. Downregulation of renal tubular Wnt/ $\beta$-catenin signaling by Dickkopf-3 induces tubular cell death in proteinuric nephropathy. Cell Death Dis. 2016;7:2155.

43. Condello S, Morgan CA, Nagdas S, Cao L, Turek J, Hurley TD, Matei D. $\beta$-Catenin-regulated ALDH1A1 is a target in ovarian cancer spheroids. Oncogene. 2015;34(18):2297-308. 